\title{
Energy currents for quasi-monochromatic fields
}

\author{
Mokhun I. I., Galushko Yu. K., Kharitonova Ye. S. and \\ Viktorovskaya Ju. Yu.
}

Chernivtsi University, 2 Kotsyubynsky St., 58012 Chernivtsi-12, Ukraine

e-mail: mokhun@itf.cv.ua

Received: 28.02 .2012

\begin{abstract}
Relations for the components of Poynting vector are obtained for quasimonochromatic waves. It is shown that the behaviour of the transverse Poynting vector component is similar to that for the coherent waves. The total angular momentum of the quasi-monochromatic wave can be separated into orbital and spin parts. Using the example of a Gaussian beam, we show that the spin angular momentum is associated with coherence characteristics of the optical beam.
\end{abstract}

Keywords: Poynting vector, angular momentum, quasi-monochromatic wave, Stokes parameters, Gaussian wave.

PACS: 42.25.-p, 42.50.Ct

UDC: 535.1

\section{Introduction}

There is no doubt that the interest to singular optics has permanently increased lately. To a great extent, this is due to attractive and promising optical applications such as optical tweezers (see, e.g., [1]). In its turn, applications of such kind have caused advanced studies of fine structure of optical fields, the characteristics of which may usually be considered as representing some specific spatial distributions. It has been shown earlier that, in the vicinity of optical singularities, the fields acquire specific values of the averaged angular momenta, which represent maxima or minima in a given area [2-4]. However, the density $j_{z}$ of such an angular momentum is only defined at the axis $\vec{r}=0$ (a 'point of applying') about which the quantity $j_{z}$ is calculated. As a result, some ambiguity appears. On the other hand, another physical quantity closely related to the angular momentum, a space distribution of characteristics of the Poynting vector (mainly its transverse component) is given by a unique function of the coordinates of each field point. Therefore investigations of behaviour of the Poynting vector defining 'energy currents' (or flows) represent an extremely important problem $[4,5]$.

It has to be noticed that the overwhelming majority of the studies performed thus far have been devoted to the monochromatic case [2-5]. In other words, the studies that deal with the angular momentum and the energy currents creating it in the polychromatic waves are at their first stage only [6-8]. At the same time, this field of researches has quite good prospects, first of all from the fundamental points of view. In our opinion, one of the crucial points is associated with the hypothesis that the angular momentum value must be somehow linked to coherence characteristics of the polychromatic waves. The existence of such a relationship seems to be obvious. It is well known that the angular momentum can be separated into its orbital and spin parts $[2,3,5]$. Then the above assumption follows at least from the fact that the spin angular momentum (see,

Ukr. J. Phys. Opt. 2012, V13, №3 
e.g., [2]) should be defined by a determinate circulation of the field vector. It would be natural that the 'level of such determinancy' should be linked to the coherence.

It is also well known that the instantaneous Poynting vector is defined by the relation (see [9])

$$
\vec{p}=\vec{E} \times \vec{H},
$$

where $\vec{E}, \vec{H}$ are the instantaneous strengths of electric and magnetic fields, respectively. As a rule, a time-averaged value of this vector is relevant in optics. A general expression for this parameter may be quite simply derived (at least, for the instantaneous Poynting vector), if we take into account the fact that the real perturbance $E_{k}(t)$ of each of the Cartesian components of the electric or magnetic fields may be represented as a Fourier integral $[10,11]$,

$$
E_{i}(t)=\int_{0}^{\infty} a(v) \cos [\varphi(v)-2 \pi v t] d v,
$$

where $i=x, y, z$. However, this cumbersome relation does not allow for carrying out simple and detailed enough analysis. The situation is essentially simplified if a quasi-monochromatic wave is considered. It is known that this wave is defined as having relatively narrow spectrum, so that (see $[9,10])$

$$
\frac{\Delta v}{\bar{v}}<<1
$$

with $\Delta v$ denoting the frequency range where $\vec{E}(t)$ is essentially different from zero and $\bar{v}$ being the mean (or the main) frequency.

In the first part of our present research we will show that, once the above assumption and the paraxial approximation are satisfied, the components of the averaged Poynting vector may be written in the form, which is very similar to that typical for completely coherent waves. After that, we will ascertain the relationship between the field angular momentum and the spectral range of the wave, using the example of a quasi-monochromatic Gaussian wave.

\section{Poynting vector components for the quasi-monochromatic field}

Let us consider a quasi-monochromatic wave, which obeys additionally the paraxial approximation. Then each of the Cartesian components $E_{i}(t)$ of the electric or magnetic fields may be represented as $[9,10]$

$$
E_{i}(t)=A_{i}(t) \cos \left[\Phi_{i}(t)-2 \pi \bar{v} t\right]
$$

where $A_{i}(t)$ and $\Phi_{i}(t)$ are slowly-varying functions when compared with the term $\cos (2 \pi \bar{v} t)$. As a consequence, the instantaneous Poynting vector may be derived under this assumption, quite similarly to strongly coherent case [4]:

$$
\left\{\begin{array}{c}
P_{x} \approx-\frac{c}{4 \pi k}\left\{E_{x} T_{2}-E_{y} T_{1}\right\} \\
P_{y} \approx-\frac{c}{4 \pi k}\left\{E_{y} T_{2}+E_{x} T_{1}\right\}, \\
P_{z} \approx \frac{c}{4 \pi}\left\{E_{x}^{2}+E_{y}^{2}\right\}
\end{array},\right.
$$

where 


$$
\left\{\begin{array}{l}
T_{1}=E_{x} \Phi_{x}^{y}-E_{y} \Phi_{y}^{x}+\frac{A_{x}^{y}}{A_{x}} E_{x, \frac{\pi}{2}}-\frac{A_{y}^{x}}{A_{y}} E_{y, \frac{\pi}{2}} \\
T_{2}=E_{x} \Phi_{x}^{x}+E_{y} \Phi_{y}^{y}+\frac{A_{x}^{x}}{A_{x}} E_{x, \frac{\pi}{2}}+\frac{A_{y}^{y}}{A_{y}} E_{y, \frac{\pi}{2}}
\end{array}\right.
$$

and

$$
\left\{\begin{array}{c}
E_{i}=A_{i}(t) \cos \left[\Phi_{i}(t)-2 \pi \bar{v} t\right] \\
E_{i, \frac{\pi}{2}}=A_{i}(t) \sin \left[\Phi_{i}(t)-2 \pi \bar{v} t\right]
\end{array} .\right.
$$

Here $A_{i}, \Phi_{i}$ are interpreted according to Eq. (4), $A_{i}^{l}, \Phi_{i}^{l}$ mean the partial derivatives respectively of $A_{i}(t)$ and $\Phi_{i}(t)$, and $i, l=x, y$. One has to note that only $x$ - and $y$-electric components appear in Eqs. (5)-(7).

Let us carry out averaging over time in Eqs. (5)-(7). One can easily show that, under our assumptions (see Eq. (3) and Eq. (4)), the following expressions hold true as a 'basis' for the averaging procedure:

$$
\left\{\begin{array}{c}
<\cos ^{2}\left(\Phi_{i}-2 \pi \bar{v} t\right)>=\frac{1}{2} \\
<\sin \left(\Phi_{i}-2 \pi \bar{v} t\right) \cos \left(\Phi_{i}-2 \pi \bar{v} t\right)>=0 \\
<\cos \left(\Phi_{i}-2 \pi \bar{v} t\right) \cos \left(\Phi_{l}-2 \pi \bar{v} t\right)>=\frac{1}{2} \cos \bar{\Delta}=\frac{1}{2} \cos \left(\bar{\Phi}_{i}-\bar{\Phi}_{l}\right) \\
<\sin \left(\Phi_{i}-2 \pi \bar{v} t\right) \cos \left(\Phi_{l}-2 \pi \bar{v} t\right)>=\frac{1}{2} \sin \bar{\Delta}=\frac{1}{2} \sin \left(\bar{\Phi}_{i}-\bar{\Phi}_{l}\right)
\end{array}\right.
$$

Here $\bar{\Delta}$ is the effective local phase difference and $\bar{\Phi}_{i}, \bar{\Phi}_{l}$ the phase functions of the components averaged over time.

After averaging Eqs. (4)-(7) we get

$$
\left\{\begin{array}{c}
\bar{P}_{x} \approx-\frac{1}{16 \pi \bar{\omega}}\left\{\left[\left(s_{0}+s_{1}\right) \bar{\Phi}_{x}^{x}+\left(s_{0}-s_{1}\right) \bar{\Phi}_{y}^{x}\right]-\frac{\partial s_{3}}{\partial y}\right\} \\
\bar{P}_{y} \approx-\frac{1}{16 \pi \bar{\omega}}\left\{\left[\left(s_{0}+s_{1}\right) \bar{\Phi}_{x}^{y}+\left(s_{0}-s_{1}\right) \bar{\Phi}_{y}^{y}\right]+\frac{\partial s_{3}}{\partial x}\right\} \\
\bar{P}_{z} \approx \frac{c}{8 \pi} s_{0}
\end{array}\right.
$$

where $s_{i}$ are the Stokes parameters and $\bar{\omega}=2 \pi \bar{\nu}$.

Thus, one can state that the notion of the averaged Poynting vector components for the quasichromatic waves is the same as that for the strongly monochromatic waves [12], with the corresponding determinate parameters. The terms in the square brackets appearing in the first and second lines of Eqs. (9) may be called as a structural or transverse orbital part of the field energy density [5]. In the coherent case, just these terms are responsible for the appearance of orbital momentum in the area of a vortex (for a scalar field) or a C-point (a point of circular polarisation) in inhomogeneously polarised fields [2, 3, 13, 14].

The last terms that appear in the expressions for the transverse components cause the spin energy currents, which define a spin angular momentum of the field. Let us take into account that the angular momentum density is given by the relation (see, e.g., [2]) 


$$
\bar{j}_{z}=\vec{r} \times \overline{\vec{P}}=\left(x \bar{P}_{y}-y \bar{P}_{x}\right) .
$$

Then one can state that, similar to the coherent case, the total angular momentum for the polychromatic beam (or, at least, for the wave with a narrow enough spectrum and in the frame of the paraxial approximation) can be separated into its orbital and spin parts.

\section{Angular momentum of quasi-chromatic Gaussian beam}

Let us repeat once again our principal statement: the angular momentum of the beam, or the angular momentum of the field area, must be related to the coherence characteristics of the wave. For instance, the spin momentum should become zero in the limiting case of absolutely depolarised wave.

As follows from the expressions for the Poynting vector components given by Eq. (9), at least the spin energy currents, and therefore the spin angular momentum associated with them, are linked to the coherence characteristics of the wave. This follows from the fact that different spectral components should contribute differently to the total angular momentum of the field. The latter situation necessarily occurs if the analysed field passes through a setup consisting of a polariser and a quarter-wave plate oriented at the angle $45^{\circ}$ with respect to the polariser axis. Let us assume that the thickness of the plate is chosen such that the spectral component with the frequency $\bar{v}$ becomes circularly polarised behind the plate. It is evident that the other spectral components would then be polarised elliptically.

Now we shall demonstrate efficiency of such a procedure for a Gaussian beam. Let the frequency spectrum of this beam correspond to the normal distribution. We notice that a presence of 'negative' frequencies in this distribution does not play an essential part, for the energy decreases rapidly when the frequency deviates from the central one. In this case the Cartesian components of the wave normalised to unity may be represented in the following form:

$$
\begin{gathered}
E_{x}=\frac{1}{\sqrt{2 \pi^{3 / 2} \sigma^{2} \sigma_{v}}} \exp \left\{-\frac{x^{2}+y^{2}}{2 \sigma^{2}}\right\} \int_{-\infty}^{\infty} \exp \left\{-\frac{(v-\bar{v})^{2}}{2 \sigma_{v}^{2}}\right\} \times \exp \left\{j\left[\Phi_{0 v}-2 \pi v t\right]\right\} d v, \\
E_{y}=\frac{1}{\sqrt{2 \pi^{3 / 2} \sigma^{2} \sigma_{v}}} \exp \left\{-\frac{x^{2}+y^{2}}{2 \sigma^{2}}\right\} \int_{-\infty}^{\infty} \exp \left\{-\frac{(v-\bar{v})^{2}}{2 \sigma_{v}^{2}}\right\} \times \exp \left\{j\left[\Phi_{0 v}-2 \pi v t+\Delta_{v}\right]\right\} d v
\end{gathered}
$$

where $\sigma$ is the 'spatial width' of the beam, $\sigma_{v}$ its 'spectral width' that defines the effective frequency band $\Delta v, \Phi_{0_{v}}$ the constant phase associated with each spectral component, and $\Delta_{v}$ the phase shift between the orthogonal components, which appears when the given spectral component passes through the quarter-wave plate. This shift may be identified via the relation

$$
\Delta_{v}=\frac{\pi}{2} \frac{v}{\bar{v}}
$$

Then the components of the Poynting vector become as follows:

$$
\left\{\begin{array}{c}
\bar{P}_{x} \approx \frac{1}{16 \pi \bar{\omega}} \frac{\partial s_{3}}{\partial y} \\
\bar{P}_{y} \approx-\frac{1}{16 \pi \bar{\omega}} \frac{\partial s_{3}}{\partial x} \\
\bar{P}_{z} \approx \frac{c}{8 \pi} s_{0}
\end{array} .\right.
$$

This is due to the fact that the phases of all the spectral components are practically constant. 
In other terms, the transverse energy currents and the angular momentum density depend only on the spatial changes in the $4^{\text {th }}$ Stokes parameter. Let us determine this Stokes parameter for our case. As shown by Born and Wolf [9], the resulting elements of the coherence matrix represent a sum of the elementary elements corresponding to each frequency $v$. As a consequence, the $4^{\text {th }}$ Stokes parameter $s_{3}$ would be given by

$$
s_{3}=j\left(J_{y x}-J_{x y}\right)=-\frac{1}{\pi \sigma^{2}} \exp \left\{-\frac{x^{2}+y^{2}}{\sigma^{2}}\right\} \exp \left(-\frac{\pi^{2} \sigma_{v}^{2}}{16 \bar{v}^{2}}\right) .
$$

Then one can write out the transverse components as

$$
\left\{\begin{array}{l}
\bar{P}_{x} \approx-\frac{1}{16 \pi^{2} \bar{\omega} \sigma^{4}} \exp \left(-\frac{\pi^{2} \sigma_{v}^{2}}{16 \bar{v}^{2}}\right) \exp \left\{-\frac{x^{2}+y^{2}}{\sigma^{2}}\right\} y \\
\bar{P}_{y} \approx \frac{1}{16 \pi^{2} \bar{\omega} \sigma^{4}} \exp \left(-\frac{\pi^{2} \sigma_{v}^{2}}{16 \bar{v}^{2}}\right) \exp \left\{-\frac{x^{2}+y^{2}}{\sigma^{2}}\right\} x
\end{array} .\right.
$$

In other words, up to the factor

$$
\alpha=\exp \left(-\frac{\pi^{2} \sigma_{v}^{2}}{16 \bar{v}^{2}}\right) \leq 1,
$$

the components of the Poynting vector turn out to be very similar to those of the monochromatic Gaussian beam [3].

As a result, the longitudinal components of the angular momentum densities and the angular momenta differ by the same factor. The magnitude of this factor determined by the width of the spectral range and the mean radiation frequency is such that it induces some decrease in the angular momentum value, when compared with the coherent case. Hence, the magnitude of the angular momentum of the quasi-monochromatic Gaussian beam is strongly linked to the wave coherence.

\section{Discussion}

Let us estimate the decrease in the angular momentum value mentioned above. It is known [15] that the indeterminancy principle for the wave processes acquires the following form:

$$
\Delta v \tau_{0} \approx 1,
$$

where $\tau_{0}$ denotes the coherence time. In the quasi-monochromatic approximation, this parameter is significantly less than the period of the wave vibration $T_{0}$,

$$
T_{0}=\frac{\bar{\lambda}}{c},
$$

where $\bar{\lambda}$ is the central wavelength from the spectral range under interest. In other words, we have

$$
\tau_{0} \sim a T_{0},
$$

where the coefficient $a$ is large enough. One can easily show that $\frac{\Delta \lambda}{\bar{\lambda}} \sim 1 / a$. In much the same way, one can obtain the relation

$$
\frac{\Delta v}{\bar{v}} \sim 1 / a
$$

now formulated in terms of $v$.

Now let us assume the $a$ value to be close to 10. In this case the spectral radiation range $\Delta \lambda$ is close to $60-70 \mathrm{~nm}$ for the 'red' visible light. This range is inherent to, e.g., light passing though interference filters and, moreover, it is close to that typical for conventional light-emitting diodes.

Ukr. J. Phys. Opt. 2012, V13, №3 
As follows from Eq. (16), the exponent determining the $\alpha$ parameter is very small $(\sim 0.001)$. Therefore the angular momentum value is practically the same as for the coherent case. The situation remains much the same even for $a=2$. It is worthwhile to notice that such small $a$ values are on the verge of any physical sense. Nevertheless, a formally calculated 'decreasing coefficient' is still as large as 0.975 . In other words, the values of the angular momentum for the quasimonochromatic Gaussian waves are practically independent of their spectral range.

\section{Conclusions}

As a result of our present study, we are able to state the following.

1. The behaviour of the transverse Poynting vector component for the quasi-monochromatic case may be defined quite similarly to the coherent case. This is why the total angular momentum of the polychromatic wave can be separated into its orbital and spin parts. This statement is true at least for the waves which meet requirements of the paraxial approximation.

2. The value of the spin angular momentum for the quasi-monochromatic wave may be associated with the coherence characteristics of the beam. It is defined only by spatial changes in the $4^{\text {th }}$ Stokes parameter.

3. The value of the angular momentum for the quasi-monochromatic Gaussian beam remains practically independent of its spectral range.

\section{Acknowledgements}

This work was supported by the Ministry of Education and Science, Youth and Sport of Ukraine under the Grant No 0111 U001161.

\section{References}

1. Lang M J and Block S M, 2003. Resource letter: LBOT-1: Laser-based optical tweezers. Amer. J. Phys. 71: 201-215.

2. Allen L, Padgett M J and Babiker M. The orbital angular momentum of light. In 'Progress in Optics' XXXIX (Ed. by E. Wolf), Elsevier Science B. V. (1999).

3. Bekshaev A, Soskin M and Vasnetsov M. Paraxial light beams with angular momentum. New York: Nova Science Publishers (2008).

4. Mokhun I. Introduction to linear singular optics. In 'Optical correlation techniques and applications‘, Chapter 1 (Ed. by O. V. Angelsky). Bellingham: SPIE Press (2007).

5. Bekshaev A, Bliokh K and Soskin M, 2011. Internal flows and energy circulation in light beams. J. Opt. 13: 053001.

6. Serna J and Movilla J M, 2001. Orbital angular momentum of partially coherent beams. Opt. Lett. 26: 405-407.

7. Angelsky O V, Gorsky M P, Maksimyak P P, Maksimyak A P, Hanson S G and Zenkova $\mathrm{C} \mathrm{Yu,} \mathrm{2011.} \mathrm{Investigation} \mathrm{of} \mathrm{optical} \mathrm{currents} \mathrm{in} \mathrm{coherent} \mathrm{and} \mathrm{partially} \mathrm{coherent} \mathrm{vector} \mathrm{fields.}$ Opt. Expr. 19: 660-672.

8. Angelsky O V, Hanson S G, Zenkova C Yu, Gorsky M P and Gorodyns'ka N V, 2009. On polarization metrology (estimation) of the degree of coherence of optical waves. Opt. Expr. 17: 15623-15634.

9. Born M and Wolf E. Principles of optics (6 ${ }^{\text {th }}$ edition). Oxford: Pergamon (1980).

10. Perina J. Coherence of light ( $2^{\text {nd }}$ edition). D. Reidel. Dordrecht (1985).

11. Nieto-Vesperinas M. Scattering and diffraction in physical optics. Wiley-Interscience Publication, John Wiley \& Sons (1981). 
12. Khrobatin R, Mokhun I and Viktorovskaya Ju, 2008. Potentiality of experimental analysis for characteristics of the Poynting vector components. Ukr. J. Phys. Opt. 9: 182-186.

13. Dennis M R, O’Holleran K and Padgett M J, 2009. Singular optics: optical vortices and polarization singularities. Prog. Opt. 53: 293-363.

14. Mokhun I and Khrobatin R, 2008. Shift of application point of angular momentum in the area of elementary polarization singularity. J. Opt. A: Pure Appl. Opt. 10: 064015.

15. Grawford F S. Waves (Berkeley Physics Course), Vol. 3. New York: McGraw-Hill Company. (1968).

Mokhun I. I., Galushko Yu. K., Kharitonova Ye. S. and Viktorovskaya Ju. Yu., 2012. Energy currents for quasi-monochromatic fields. Ukr.J.Phys.Opt. 13: 151 - 157.

Анотація. Отримані співвідношення для компонент вектора Пойнтинга для квазі монохроматичної хвилі. Показано, щзо поведінка поперечної компоненти вектора Пойнтинга $\epsilon$ подібною до поведінки у випадку когерентних хвиль. Загальний кутовий момент квазі монохроматичної хвилі може бути розділеним на орбітальний і спіновий моменти. Використовуючи приклад гаусівського променя ми показали, щяо спіновий кутовий момент асоціюється з когерентними характеристиками оптичного променя. 